\title{
Search for Ultra High Energy Cosmic Rays from Space - The JEM-EUSO Program
}

\author{
M. Bertaina*1, for the JEM-EUSO Collaboration ${ }^{\dagger}$ \\ ${ }^{1}$ Department of Physics, University of Torino, Italy \\ E-mail: bertainadto.infn.it
}

\begin{abstract}
The origin and nature of Ultra-High Energy Cosmic Rays (UHECRs) remain unsolved in contemporary astroparticle physics. To give an answer to these questions is rather challenging because of the extremely low flux of a few per $\mathrm{km}^{2}$ per century at extreme energies (i.e. E $>5 \times 10^{19}$ $\mathrm{eV})$. The central objective of the JEM-EUSO program, Joint Experiment Missions for Extreme Universe Space Observatory, is the realisation of an ambitious space-based mission devoted to UHECR science. A super-wide-field telescope will look down from space onto the night sky to detect UV photons emitted from air showers generated by UHECRs in the atmosphere. The JEMEUSO program includes several missions from ground (EUSO-TA), from stratospheric balloons (EUSO-Balloon, EUSO-SPB1, EUSO-SPB2), and from space (TUS, Mini-EUSO) employing fluorescence detectors to demonstrate the UHECR observation from space and prepare the large size missions K-EUSO and POEMMA. We review the scientifical objectives associated with the developing projects of the JEM-EUSO program and the technological achievements allowing them.
\end{abstract}

36th International Cosmic Ray Conference -ICRC2019-

July 24th - August 1st, 2019

Madison, WI, U.S.A.

\footnotetext{
${ }^{*}$ Speaker.

${ }^{\dagger}$ for collaboration list see PoS(ICRC2019)1177
} 


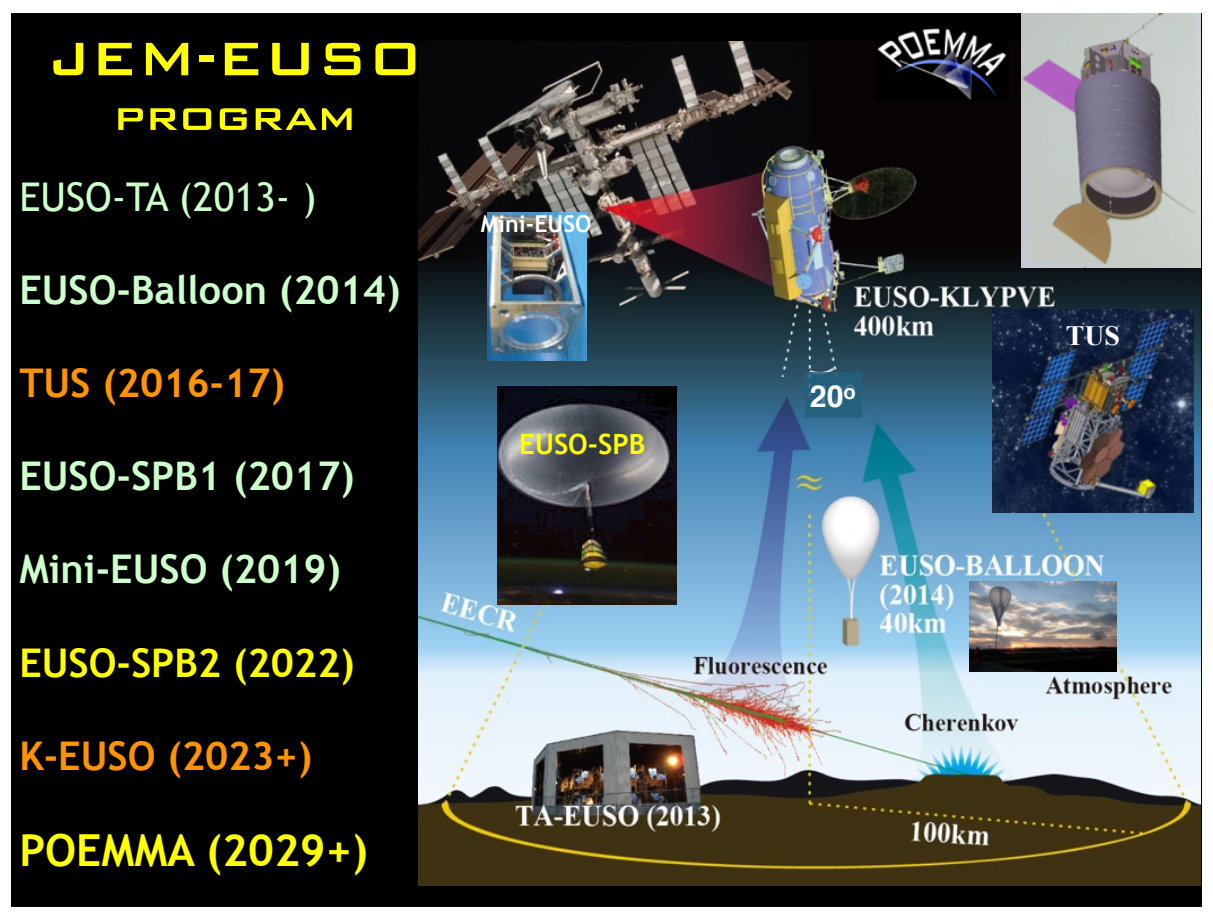

Figure 1: Roadmap of the JEM-EUSO program. See text for details.

\section{Introduction}

The main objective of the JEM-EUSO program [1], Joint Experiment Missions for Extreme Universe Space Observatory, is the realization of an ambitious space-based mission devoted to scientific research of UHECRs [2]. The JEM-EUSO program includes several missions from ground (EUSO-TA [3]), from stratospheric balloons (EUSO-Balloon [4], EUSO-SPB1 [5], EUSOSPB2 [6]), and from space (TUS [7], Mini-EUSO [8]) employing fluorescence detectors to demonstrate the UHECR observation from space and prepare the large size missions K-EUSO [9] and POEMMA [10]. The timeline of the different projects is shown in Fig. 1, while the key results obtained so far are summarized in [11].

\section{The ground-based telescope EUSO-TA}

EUSO-TA is a ground-based telescope, installed at the TA site in Black Rock Mesa, Utah, USA. This is the first detector to successfully use a Fresnel lens based optical system and MultiAnode Photomultipliers (MAPMT). Each MAPMT has 64 channels, and they are grouped in blocks of $2 \times 2$ to form one Elementary Cell (EC). Nine ECs form one Photo-Detector Module (PDM) which has, therefore, 2304 channels encompassing a $10.6^{\circ} \times 10.6^{\circ}$ field of view (FoV) for the detection of UHECRs. The telescope is located in front of one of the fluorescence detectors of the TA experiment. Since its first operation in 2015, a few campaigns of joint observations allowed EUSO-TA to detect several UHECRs events as well as meteors. The limiting magnitude of 5.5 on summed frames $(\sim 3 \mathrm{~ms})$ has been established. Measurements of the UV night sky emission 
in different conditions and moon phases have been performed. These observations provided important data to optimise the detector technology in view of the upcoming space-based missions. A detailed description of the results of EUSO-TA and the prospects of future upgrades of the detector are described in [12]. Regarding the performance, 9 UHECR events were identified so far during 120 hours of UHECR-dedicated observations which can be directly compared with simulations to determine the energy threshold of the experiment (see [13]). The resulting information is also useful for the upcoming JEM-EUSO balloon and space missions. The detector can also be calibrated using stars in the FoV, as discussed in [14]. The future upgrades of the detector include a new acquisition system based on Zynq board and the implementation of a self-triggering system already in use for the balloon missions [15], in order to obtain a detailed characterisation of the UHECR detection performances. Moreover, high sensitivity CMOS cameras will be used on the same site to detect meteoroids and search for nuclearites [16] in parallel to EUSO-TA instrumentation.

\section{The stratospheric balloon program: EUSO-Balloon, EUSO-SPB1 \& EUSO-SPB2}

The JEM-EUSO program includes three stratospheric balloon missions with increasing level of performance and upgraded designs. In addition to demonstrating the capabilities of the JEMEUSO instruments to detect and reconstruct showers from the edge of space, they also give access to direct measurement of the UV nightglow emission and artificial UV contributions above ground and oceans, which are important information to optimise the design of the space-based missions. Two balloon flights have been performed so far: EUSO-Balloon (Canada, 1 night) and EUSOSPB1 (Pacific Ocean, 12 nights). A third one (EUSO-SPB2) is under preparation to fly in 2022 from Wanaka, New Zealand.

EUSO-Balloon [4] was launched by CNES from the Timmins base in Ontario (Canada) on the moonless night of August 25, 2014 UT. After reaching the floating altitude of $\sim 38 \mathrm{~km}$, EUSOBalloon imaged the UV intensity in the wavelength range $290-430 \mathrm{~nm}$ for more than 5 hours before descending to ground. The refractor telescope consisted of a similar apparatus as EUSOTA. The spatial and temporal resolutions of the detector were $130 \mathrm{~m}$ and $2.5 \mu \mathrm{s}$, respectively. The full FoV in nadir mode was $\sim 11^{\circ}$. During 2.5 hours of EUSO-Balloon flight, a helicopter circled under the balloon operating UV flashers and a UV laser to simulate the optical signals from UHECRs, to calibrate the apparatus, and to characterise the optical atmospheric conditions. During flight EUSO-Balloon took more than 2.5 million images that have been analysed to infer different information: study of the performance of the different parts of the detector; response of the detector to the UV flasher and laser events; UV radiance from the Earth atmosphere and ground in different conditions: clear and cloudy atmosphere, forests, lakes, as well as city lights. The measurement of UV light intensity is relevant for a JEM-EUSO-like mission as it is one of the key parameters to estimate the exposure curve as a function of energy [17]. The results of this analysis are reported in [18] and are in the band of previous measurements confirming a good understanding of the detector performance also in this respect, which is very important in view of a space-based observatory like JEM-EUSO. The helicopter events proved to be extremely useful to understand the system's performance and to test the capability of EUSO-Balloon to detect and reconstruct signals similar to Extensive-Air-Shower (EAS) events. Laser tracks were used to test the reconstruction algorithms [19]. The data collected by EUSO-Balloon were used together with those collected by 
EUSO-TA and experiments as TurLab to define an internal trigger logic [20] that was implemented for the EUSO-SPB1 flight.

EUSO-SPB1 [5] was launched April 252017 from Wanaka New Zealand as a mission of opportunity on a NASA SPB test flight planned to circle the southern hemisphere. One of the scientific goals was to make the first observation of UHECR-EASs by looking down on the atmosphere with an optical fluorescence detector from the near space altitude of $33 \mathrm{~km}$. Unfortunately, although the instrument was showing nominal behaviour and performances, the flight was terminated prematurely in the Pacific Ocean about $300 \mathrm{~km}$ SE of Easter Island after only 12 days 4 hours aloft, due to a leak in the carrying balloon. The telescope was an upgraded version of that used in the EUSO-Balloon mission. In particular, a SiPM camera was installed [21]. An autonomous internal trigger was implemented according to [20] to detect UHECRs. Prior to flight, in October 2016, the fully assembled EUSO-SPB1 detector was tested for a week at the EUSO-TA site to measure its response and to calibrate it by means of a portable Ground Laser System (GLS). Observations of Central Laser Facility (CLF), stars, meteors were performed. The $\sim 50 \%$ trigger efficiency was reached at laser energies whose luminosity is equivalent to $\sim 45^{\circ}$ inclined EAS of $\mathrm{E} \sim 3 \times 10^{18} \mathrm{eV}$ seen from above by a balloon flying at $33 \mathrm{~km}$ altitude. During flight, $\sim 30$ hours of data were collected, the trigger rate was tipycally a few $\mathrm{Hz}$, which is compliant with JEMEUSO requirements [22]. A deep analysis of the collected data has been performed. Tracks of CRs directly crossing the detector have been recognized. However, no EAS track has been clearly identified $[23,24]$. Simulations post-flight indicate that the number of expected events is $\sim 1$ in the available data sample indipendently of the balloon height and UV level confirming pre-flight expectations for such a flight duration [25]. The role of clouds has been extensively studied and the exposure reduces to $\sim 0.6[26,27]$ taking them into account. Data taken by the IR camera of EUSOSPB1 are being analysed to test methods developed in the past [28] to retrieve the cloud-top-height in the frame of the JEM-EUSO mission and to estimate the cloud fraction [29].

The subsequent step of the JEM-EUSO program development is currently under realization: EUSO-SPB2 [30]. It will be equipped with 2 telescopes. One telescope will be devoted to UHECR measurements using the fluorescence technique. The Focal Surface (FS) [31] will be equipped with 3 PDMs to increase the UHECR collection power. A more performing optics (Schmidt camera) and a shorter temporal resolution of $1 \mu$ s will be used to lower the energy threshold of the instrument. The FS of the other telescope will be based on SiPMT sensors and a dedicated electronics to detect the Cherenkov emission in air by UHECR-generated EASs [32]. In perspective they will test the capability to detect EAS generated by $v_{\tau}$ interacting in the Earth crust [33]. For this observation the detector will be pointing slightly below the limb. The observation above the limb will allow to study UHECRs through their Cherenkov emission [34, 35]. EUSO-SPB2 is programmed to fly by 2022 from Wanaka, New Zealand on a NASA Super Pressure Balloon.

\section{The precursor space-based missions: TUS and Mini-EUSO}

The Track Ultraviolet Setup (TUS) detector was launched on April 28, 2016 as a part of the scientific payload of the Lomonosov satellite. TUS [7] is the world's first orbital detector aiming at detecting UHECRs. The instrument was actively recording data till November 2017. Different scientific modes were tested: cosmic ray, lightning and meteor modes. The satellite has a sun- 
synchronous orbit with an inclination of $97.3^{\circ}$, a period of $\sim 94 \mathrm{~min}$, and a height of $470-500$ $\mathrm{km}$. The telescope consists of two main parts: a modular Fresnel mirror-concentrator with an area of $\sim 2 \mathrm{~m}^{2}$ and 256 PMTs arranged in a $16 \times 16$ photo-receiver matrix located in the focal plane of the mirror. The pixel's FoV is $10 \mathrm{mrad}$, which corresponds to a spatial spot of $\sim 5 \mathrm{~km} \times 5 \mathrm{~km}$ at the sea level from a $500 \mathrm{~km}$ orbit height. Thus, the full area observed by TUS at any moment is $\sim 80 \mathrm{~km} \times 80 \mathrm{~km}$. TUS is sensitive to the near UV band and in cosmic ray mode has a time resolution of $0.8 \mu \mathrm{s}$ in a full temporal interval of 256 time steps. TUS data offer the opportunity to develop strategies in the analysis and reconstruction of events which will be essential for future space-based missions such as K-EUSO. During its operation TUS has detected about $8 \times 10^{4}$ events that have been subject to an offline analysis to select among them those satisfying basic temporal and spatial criteria of UHECRs. A few events passed this first screening. One specific event registered in perfect observational conditions was deeply scrutinized. Its phenomenology and the possible interpretations are reported in detail in [36]. In order to perform a deeper analysis of such candidates, a dedicated algorithm to reconstruct the arrival direction in TUS events and a specific version of ESAF [37] are being developed. The data acquired in meteor mode are being analyzed to search for slow events such as nuclearites. Simulations indicate that nuclearites can be detected in this mode [38], and thanks to the gigantic area that can be monitored by such space-based detectors, these telescopes have the potential to set search yields better than the constraints given by former experiments.

While TUS was conceived mainly to prove the observation of UHECRs from space with a FS-instrumentation similar to ground-based detectors, Mini-EUSO has been developed in order to test the same FS-instrumentation foreseen for K-EUSO and POEMMA. Mini-EUSO [39] is a UV telescope to be placed in August 2019 inside the ISS, looking down on the Earth from a nadir-facing window in the Russian Zvezda module. Mini-EUSO will map the Earth in the UV range (290 - 430 $\mathrm{nm}$ ) with a spatial and temporal resolutions of $\sim 6 \mathrm{~km}$ (similar to TUS) and $2.5 \mu \mathrm{s}$, respectively. Mini-EUSO has a FS similar to EUSO-TA, but with a different acquisition system [40] . The optical system consists of 2 Fresnel lenses of $25 \mathrm{~cm}$ diameter with a large FoV of $\sim 22^{\circ}$. A multiple level trigger will allow the measurement of UV transients at different time scales, complementing TUS observations. Laboratory experiments at TurLab [41] as well as open-sky data acquistions with Mini-EUSO engineering model [42] and simulations confirm the expected sensitivity of MiniEUSO to different light phenomena and to EAS-like transients around $10^{21} \mathrm{eV}$. It is planned to use a ground-based UV laser system to test such sensitivity [43]. Mini-EUSO has also other scientific objectives, among them the study of Transient Luminous Events (TLEs), meteors, and the search for stranglets and detection of space debris [44, 45].

\section{The target space-based missions of the program: K-EUSO \& POEMMA}

The central objective of K-EUSO [9] is the first consistent measurement of the UHECR flux over the entire sky with unprecedented and almost uniform exposure. K-EUSO is a result of the joint efforts to improve the performance of the Russian KLYPVE mission [46], by employing the technologies developed for the JEM-EUSO mission, such as the focal surface detectors and the readout electronics. Since its first conception as KLYPVE, K-EUSO project has passed various modifications aimed to increase FoV and UHECR statistics. It will be the first detector with a real 
capability for UHECR spectrum and anisotropy study with a yearly exposure of $\sim 4$ times Auger and a flat full celestial sphere coverage. The adopted optical layout is a Schmidt camera covering a FoV of 40 degree with an entrance pupil diameter of $2.5 \mathrm{~m}$, a $4 \mathrm{~m}$ diameter spherical mirror and a focal length of $1.7 \mathrm{~m}$. The temporal (sampling time is $1 \mu \mathrm{s}$ ) and spatial (angular resolution per pixel $0.066^{\circ}$ ) evolution of UV light recorded by K-EUSO will allow the reconstruction of the EAS, with sufficient energy and arrival direction resolutions. The camera focal plane consists of 52 PDMs like in EUSO-SPB2, for a total of $1.2 \times 10^{5}$ pixels. A pixel will cover $\sim 0.8 \mathrm{~km}$ on the surface of the Earth for ISS altitude of $400 \mathrm{~km}$. Attached to the Russian MRM-1 module on-board ISS, K-EUSO is planned to operate for minimum of 2 years and it can function more than 6 years if the lifetime of the ISS is extended.

The Probe Of Extreme Multi-Messenger Astrophysics (POEMMA) mission [10] is being designed to establish charged particle astronomy with UHECRs and to observe cosmic neutrinos from space. POEMMA will monitor colossal volumes of the Earth's atmosphere to detect extensive air showers (EASs) produced by extremely energetic cosmic messengers: cosmic neutrinos above 20 PeV and UHECRs above $20 \mathrm{EeV}$ over the entire sky. The POEMMA design combines the concept developed for the OWL mission [47] and the experience of the JEM-EUSO fluorescence detection camera. POEMMA is composed of two identical satellites flying in formation at $525 \mathrm{~km}$ altitude with the ability to observe overlapping regions during moonless nights at angles ranging from Nadir to just above the limb of the Earth, but also with independent pointing strategies to exploit at maximum the scientific program of the mission [48]. Each POEMMA satellite consists of a 4-meter photometer designed with Schmidt wide $\left(45^{\circ}\right)$ FoV optics. The POEMMA FS is composed of a hybrid of two types of cameras: about $90 \%$ of the FS is dedicated to the POEMMA fluorescence camera (PFC), while POEMMA Cherenkov camera (PCC) occupies the crescent moon shaped edge of the FS which images the limb of the Earth. The PFC is composed of JEM-EUSO PDMs based on MAPMTs. The typical time between images for the PFC is about $1 \mu \mathrm{sec}$. The much faster POEMMA Cherenkov camera (PCC) is composed of Silicon photo-multipliers (SiPMs) also flown in EUSO-SPB1 and soon to be tested in space with Mini-EUSO. The PFC registers UHECR tracks from Nadir to just below the Earth's limb, while the PCC registers light within the Cherenkov emission cone of up-going showers around the limb of the Earth and also from high energy cosmic rays above the limb of the Earth.

\section{Acknowledgments}

This work was partially supported by Basic Science Interdisciplinary Research Projects of RIKEN and JSPS KAKENHI Grant (JP17H02905, JP16H02426 and JP16H16737), by the Italian Ministry of Foreign Affairs and International Cooperation, by the Italian Space Agency through the ASI INFN agreement n. 2017-8-H.0 and contract n. 2016-1-U.0, by NASA award 11-APRA0058 in the USA, by the Deutsches Zentrum für Luft- und Raumfahrt, by the French space agency CNES, the Helmholtz Alliance for Astroparticle Physics funded by the Initiative and Networking Fund of the Helmholtz Association (Germany), by Slovak Academy of Sciences MVTS JEMEUSO as well as VEGA grant agency project 2/0132/17, by National Science Centre in Poland grant (2015/19/N/ST9/03708 and 2017/27/B/ST9/02162), by Mexican funding agencies PAPIIT-UNAM, CONACyT and the Mexican Space Agency (AEM). Russia is supported by ROSCOSMOS and the 
Russian Foundation for Basic Research Grant No 16-29-13065. Sweden is funded by the Olle Engkvist Byggmästare Foundation.

\section{References}

[1] M. Ricci (JEM-EUSO Coll.), J. Phys. Conf. Ser. 718 (2016) no.5, 052034.

[2] J.H. Adams et al (JEM-EUSO Coll.), Experimental Astronomy 40 (2015) 3.

[3] G. Abdellaoui et al (JEM-EUSO Coll.), Astroparticle Physics 102 (2018) 98.

[4] J.H. Adams et al (JEM-EUSO Coll.), Experimental Astronomy 40 (2015) 281.

[5] L. Wiencke and A. Olinto for the JEM-EUSO Coll., PoS(ICRC2017), 1097.

[6] J.H. Adams et al, ArXiv e-prints [[arXiv]1703.04513 (2017).

[7] P. Klimov et al (TUS Coll.), Space Science Reviews 8 (2017) 1.

[8] F. Capel et al, Advances in Space Research, 62 (2018) 2954.

[9] M. Casolino M. et al (JEM-EUSO Coll.), PoS(ICRC2017) 368.

[10] A. Olinto et al (POEMMA Coll.), PoS(ICRC2017) 542.

[11] F. Fenu et al (JEM-EUSO Coll.), Results from the first missions of the JEM-EUSO program, PoS(ICRC2019) 251.

[12] L. Piotrowski et al (JEM-EUSO Coll.), Results and status of the EUSO-TA detector, PoS(ICRC2019) 388.

[13] F. Bisconti et al (JEM-EUSO Coll.), EUSO-TA ground based fluorescence detector: analysis of the detected events, PoS(ICRC2019) 197.

[14] Z. Plebaniak et al (JEM-EUSO Coll.), Calibration of EUSO-TA detector with stars, PoS(ICRC2019) 393.

[15] M. Battisti et al (JEM-EUSO Coll.), EUSO-TA \& EUSO-SPB2 trigger logic, PoS(ICRC2019) 426.

[16] R. Ide et al, Study of Fast Moving Nuclearites and Meteoroids using High Sensitivity CMOS Camera, PoS(ICRC2019) 525.

[17] J.H. Adams et al (JEM-EUSO Coll.), Astroparticle Physics 44 (2013) 76.

[18] G. Abdellaoui et al (JEM-EUSO Coll.), Astroparticle Physics 111 (2019) 54.

[19] G. Abdellaoui et al (JEM-EUSO Coll.), J. of Instrumentation 13 (2018) 05023.

[20] G. Abdellaoui et al (JEM-EUSO Coll.), Nucl. Instr. \& Meth. A 866 (2017) 150.

[21] W. Painter et al (JEM-EUSO Coll.), Silicon Photomultipliersfor Orbital Ultra High Energy Cosmic Ray Observation, PoS(ICRC2019) 285.

[22] M. Battisti et al (JEM-EUSO Coll.), Nucl. Instr. \& Meth. A 936349 (2019).

[23] A. Diaz et al (JEM-EUSO Coll.), EUSO-SPB1: Flight data classification and Air shower search results, PoS(ICRC2019) 240.

[24] M. Vrabel et al (JEM-EUSO Coll.), Machine Learning Approach for Air Shower Recognition in the EUSO-SPB Experiment Data, PoS(ICRC2019) 456.

[25] J. Eser et al (JEM-EUSO Coll.), EUSO-SPB1 overview, PoS(ICRC2019) 247. 
[26] K. Shinozaki et al (JEM-EUSO Coll.), An estimation of the exposure of air shower detectionby the EUSO-SPB1 mission, PoS(ICRC2019) 427.

[27] S. Monte et al (JEM-EUSO Coll.), WRF and radiative methods for Cloud Top Height retrieval along EUSO-SPB1 trajectory, PoS(ICRC2019) 455.

[28] A. Anzalone et al, IEEE Trans. Geoscience \& Remote Sensing 57304 (2019).

[29] A. Bruno et al (JEM-EUSO Coll.), A method for Cloud Mapping in the Field of View of the Infra-Red Camera during the EUSO-SPB1 flight, PoS(ICRC2019) 454.

[30] L. Wiencke et al (JEM-EUSO Coll.), The Extreme Universe Space Observatory on a Super-Pressure Balloon II Mission, PoS(ICRC2019) 466.

[31] V. Scotti et al (JEM-EUSO Coll.), The Data Processor of the EUSO-SPB2 telescopes, PoS(ICRC2019) 368.

[32] N. Otte et al (JEM-EUSO Coll.), Development of a Cherenkov Telescope for the Detection of Ultra-High Energy Neutrinos with EUSO-SPB2 and POEMMA, PoS(ICRC2019) 977.

[33] A. Cummings et al, A More Complete Phenomenology of Tau Lepton Induced Air Showers, PoS(ICRC2019) 862.

[34] J. Szabelski et al (JEM-EUSO Coll.), Cosmic Ray Mass Composition around $10^{18} \mathrm{eV}$ with Horizontal Cherenkov EAS Light Balloon Measurements, PoS(ICRC2019) 433.

[35] K. Krolik et al (JEM-EUSO Coll.), Cherenkov light from horizontal Air Showers, PoS(ICRC2019) 321.

[36] M. Zotov et al (TUS Coll.), An UHECR-like event registered with the TUS orbital detector, PoS(ICRC2019) 193.

[37] F. Fenu et al (JEM-EUSO Coll.), Simulations for the JEM-EUSO program with ESAF PoS(ICRC2019) 252.

[38] K. Shinozaki et al (TUS Coll.), Search for slow-moving nuclearite-induced events using the TUS orbital UHECR detector PoS(ICRC2019) 545.

[39] M. Casolino et al (JEM-EUSO Coll.), The MINI-EUSO mission to study UV emissions from the International Space Station, PoS(ICRC2019) 212.

[40] A. Belov et al, Advances in Space Research, 62 (2018) 2966.

[41] H. Miyamoto et al (JEM-EUSO Coll.), The EUSO@Turlab Project: Tests of Mini-EUSO Engineering Model, PoS(ICRC2019) 194.

[42] F. Bisconti et al (JEM-EUSO Coll.), Mini-EUSO engineering model: tests in open-sky condition, PoS(ICRC2019) 198.

[43] V. Kungel et al (JEM-EUSO Coll.), UV laser system test of Mini-EUSO, PoS(ICRC2019) 325.

[44] T. Ebisuzaki et al., Acta Astronautica 112 (2015) 102.

[45] H. Miyamoto et al (JEM-EUSO Coll.), Space debris detection and tracking with the techniques of cosmic ray physics, PoS(ICRC2019) 253.

[46] B. Khrenov et al., Nuclear Physics B Proceedings Supplements, 113/1 (2002) 115.

[47] F.W. Stecker et al, Nucl. Phys. B 136C (2004) 433.

[48] A. Olinto et al (POEMMA Coll.), POEMMA: Probe Of Extreme Multi-Messenger Astrophysics, PoS(ICRC2019) 378. 